\title{
Customer Relationship Management: An IT Success as Multifunctional Domain and it's Fututre Directions
}

\author{
Deepak A. Vidhate, PhD \\ Prof. \& Head, Department of \\ Information Technology \\ Dr. Vitthalrao Vikhe Patil College of \\ Engineering \\ Ahmednagar, India
}

\author{
Mansi Palve \\ Department of Information \\ Technology \\ Dr. Vitthalrao Vikhe Patil College of \\ Engineering \\ Ahmednagar, India
}

\author{
Anushka Joshi \\ Department of Information \\ Technology \\ Dr. Vitthalrao Vikhe Patil College of \\ Engineering, \\ Ahmednagar, India
}

\author{
Aishwarya Lonkar \\ Department of Information \\ Technology \\ Dr. Vitthalrao Vikhe Patil College of \\ Engineering \\ Ahmednagar, India
}

\author{
Tushar Nimase \\ Department of Information \\ Technology \\ Dr. Vitthalrao Vikhe Patil College of \\ Engineering \\ Ahmednagar, India
}

\author{
Harshavardhini Mane \\ Department of Information \\ Technology \\ Dr. Vitthalrao Vikhe Pati College of \\ Engineering \\ Ahmednagar, India
}

\author{
Adhikar Shinde \\ Department of Information Technology \\ Dr. Vitthalrao Vikhe Patil College of Engineering \\ Ahmednagar, India
}

\author{
Dheeraj Kohli \\ Aux Services, Cherrywood Trail, \\ Coppell, TX, USA
}

\begin{abstract}
Customer Relationship Management (CRM) is a business strategy for improving profitability by focusing on customer needs and creating an attentive relationship with the customer. It's an approach that focuses on managing relationships between the company and the customer and how to develop and retention it. A CRM system can provide powerful competitive advantage for organizations to enable them to survive in today's market. There are various complex organizations, offering a wide range of services, which involve a multiplicity of customers, stakeholders and service providers; both in terms of type and number. Satisfying a diverse set of customer groups is complex, and requires development of strategic Customer Relationship Management (CRM). This paper contributes to the multifunctional domain area, by proposing an approach that scopes CRM strategy, allowing us a better understanding CRM implementation in diverse area; maximizing alignment of customer and management desires, expectation and needs. In IT terms, CRM means an enterprise - wide integration of technologies working together such as data warehouse, web site, phone support system, accounting, sales, marketing and production. This paper is directed towards information technology (IT) and marketing management (CRM) solution. The goal of this article is not to provide at all inclusive tutorial on CRM, but rather to provide a high level insight of the fundamental principles behind CRM and critical aspects of the IT development process.
\end{abstract}

\section{Keywords}

Customer relationship management, stakeholders, multifunctional domain, marketing management solution

\section{INTRODUCTION}

If you have never heard about the importance of Customer Relationship Management (CRM) consider this: Forbes, an American business magazine, refer to an enterprise software forecast from Gartner (one of the biggest technology analysts and consultant companies in the world) indicating that CRM will have the lead in growth in all enterprise software categories and furthermore they predict a worldwide spending of 36.5 billion dollars in 2017 into CRM technologies (Gartner, 2013). CRM is growing at fast pace, carried forward with the rise of new communication technologies. CRM implementation is actually sector-independent. Having a sales process is the only pre-requisite. The CRM simply optimizes the same for maximum efficiency. The CRM approach has received increased attention as a marketing concept during the last decades, both amongst practitioners and in academia (c.f., for instance, [Sin et al. 2005; Osarenkhoe and Bennani 2007; Wilson et al. 2002]). The number of articles and books on CRM appears to be increasing incrementally, and the implication from this is that a new sub-discipline of marketing research is emerging. As with most emerging research fields, there appears to be a certain amount of confusion associated with CRM research, not least since there is a great variety of topics that have been addressed in CRM research. There is a need for reflection on this emerging research field, and consideration must be given to identifying trends and relevant topics for further research. The main purpose of this paper is to add to the image of CRM research by focussing on identifying trends and topics focused, using the perspective of CRM when it is divided into four separate branches, namely, Strategic CRM, Analytical CRM, Operational CRM, and Collaborative CRM. In addition to this, the purpose is to identify under-researched areas within the body of CRM research that suggest that there are research questions to be 
addressed by means of further research. Bain \& Company (2013) is one of many firms of consultants that representing the system of CRM and they describe it as a process companies use to understand their customer groups and respond quickly - and at times, instantly - to shifting customer desires. CRM technology allows firms to collect and manage large amounts of customer data and carry out strategies based on that information. CRM data also provide companies with important new insights into customers' needs and behavior, allowing them to tailor products to targeted customer segments. In short, a system of CRM is a system based on the company's customers and how the company can provide the best service and product for this customer. The system will focus on the relationship and the aim is to gain loyalty and long-term customers.

\section{LITERATURE REVIEW}

Goldenberg (2000) believes that CRM is not merely technology applications for marketing, sales and services but rather when it is successfully implemented; it enables firms to have crossfunctional, customer-driven, technology-integrated business process management strategy that maximses relationships. Chin et al (2003) stated that that due to many technological solutions available for CRM automation, it is often misconstrued as a piece of technology. But they maintained that in recent times many companies have realized the strategic importance of CRM, and as a result, it is becoming a business value-effort rather than technologycentric effort.

According to Greenberg (2004), CRM generally is an enterprise-focused endeavor encompassing all departments in a business . He further explains that, in addition to customer service, CRM would also include, manufacturing, product testing, assembling as well as purchasing, and billing, and human resource, marketing, sales and engineering.

Like any other new function, CRM too has its own drawbacks and challenges. Any organization that seeks to implement CRM may focus on value creation and on a continuous stream of profits. They will give up their myopic fix that CRM is the fixed responsibility of marketing or IT department. The firms will realize that in order for CRM to contribute to corporate renaissance, the CRM responsibility must rise to the level of CEO.

CRM will be more strategy driven, and thus be able to concentrate on what customer expects from relationships. The 'final take' for the CEOs will be that CRM is and can be a vehicle for cultural integration in the organization. In short, a true CRM encourages a relationship view of the world that goes beyond the customers, includes multi -members and facilitates corporate renaissance.

Two trends have brought CRM to the forefront, explains Boston University professor Tom Davenport, who directs Andersen consulting's Institute for Strategic Change. First, as global competition has increased and products have become harder to differentiate, "companies have begun moving from a product-centric view of the world to a customer-centric one," says Davenport.

Second, technology has ripened to the point where it is possible to put customer information from all over the enterprise into a single system. "Until recently, it didn't have the ability to manage the complex information about customers, because information was stored in 20 different systems," says Davenport. But as network and Internet technology has matured, CRM software has found its place in the world. Many companies are turning to customerrelationship management systems to better understand customer wants and needs. CRM applications, often used in combination with data warehousing, E-commerce applications, and call centers, allow companies to gather and access information about customers' buying histories, preferences, complaints, and other data so they can better anticipate what customers will want. The goal is to instill greater customer loyalty.

\section{PERSPECTIVE OF CRM BRANCHES}

The image of CRM research by focusing on identifying trends and topics focused, using the perspective of CRM when it is divided into four separate branches, namely, Strategic CRM, Analytical CRM, Operational CRM, and Collaborative CRM.

\section{A. Strategic CRM}

One branch of CRM is to regard it as an enterprise wide strategy in which the main focus is on the customer. It is also assumed that, within an enterprise, it is a core managerial task to champion and implement this focus as a CRM strategy, including placing an emphasis on the systematic analysis and use of customer information as a platform for marketing and management. Strategic CRM is, in a close call with Analytic CRM, the largest research field according to the literature review. Since strategy is by definition a complex phenomenon, articles about Strategic CRM also discuss many topics.

- Among the major issues are the CRM paradigm; the customer-centric view and customer focus. Long-term customer relations and value creation, together with a focus on the relationship with the individual customer, also constitute major parts of the CRM paradigm [Sin et al. 2005].

- The implementation failures mentioned form the backdrop to research articles dealing with the introduction of CRM systems and strategies. The implementation of a CRM approach is recognised as being a complex process, full of problems and barriers that must be managed in a proper manner in order to promote the success of an implementation. Altogether, it is a question of change management in order to impact processes, people, supplier/outsource relationships and technology. Organisational cultures may have to be changed, and a readiness for change has to be achieved. [Chen and Popovich 2003; Peppard 2000; Ryals and Knox 2001].

- One key factor in an implementation context is the commitment from managers [Bull 2003; Osarenkhoe and Bennani 2007], a factor which is often discussed when dealing with other success factors. Without managerial commitment, any implementation of CRM strategies is thought to be deemed as a failure [Chen and Popovich 2003] Top management commitment and the need for a human resource management (HRM) in line with a customer focus is basic to the CRM approach. Closely related to this is the management of an organisation culture that embraces the saliency of a customer focus in operations [Boulding et al. 2005; Teo et al. 2006; Wilson 2005].

- Furthermore, CRM cannot be implemented without proper support from information systems and architecture. The requirement for integration increases for different IT based processes within the enterprise. Initiatives such as e-business, and electronic services in general, open up and make many of the organisation's previously internal information systems transparent to the customer. The Enterprise Resource Planning (ERP) system provides the heart of information for business processes, Supply Chain Management (SCM) integrates suppliers with customers and Decision Support Systems (DSS) provide a foundation for decisions. Altogether there is a need for the integration of all applications across the enterprise, in addition to a requirement for a common architecture supporting the business. A current trend is Enterprise Application Integration (EAI) whose ultimate goal is to integrate all applications within the enterprise into a coherent whole [Themistocleus et al. 2005; Puschmann and Alt 2001]. 
Such integration offers the possibility to support an enterprise wide strategy based upon integration and customer focus.

- Other topics discussed at a strategic level are new business practices, such as e-commerce, m-commerce (mobile), and multichannel management in general, i.e. strategies for deploying CRM in new or all channels, and the management of the channels. (Payne and Frow 2004)

\section{B. Analytical CRM}

The customer knowledge data base formed from the systematic collecting and storing of customer data is perceived as an asset to the enterprise. Through an analysis of the data in this data base it is possible to improve marketing efficiency in different ways. It is important that the ICT development has made it possible to both gather and analyse customer data in a much more systematic and efficient way than was previously the case before the computer revolution. Analytical CRM is, according to the literature review (see Table 1), almost as popular as Strategic CRM.

- The field is dominated by the concept of data mining. The essence of data mining is to analyse large quantities of data in order to discover meaningful patterns and relationships.

- Another major topic within Analytic CRM is predictive modelling. The purpose is to build models for the prediction of purchase behaviour and for purchase forecasting [Xu and Walton 2005].

- The goal of prediction is twofold: The first goal is to maintain customer loyalty in order to maximise customer retention (antichurn) (c.f. [Van den Poel and Lariviere 2004; Verhoef et alt. 2001]). Another goal is customer segmentation, through which customers are selected and differentiated in to personalise and add value to offerings. Value is also added for a company that is able to improve cross-selling [Verhoef 2001].

- The development and testing of methods for customer data analysis appear to be attractive topics suitable for statistical and mathematical approaches. Common statistical methods are used for this purpose, including regression analysis, but also more advanced methods, e.g. neural networks, signalling game, Baysean equilibrium, Walraisan general equilibrium approach, Markov chain transition matrix, text analysis, fuzzy clustering, and novelty detection. [Burez and Van den Poel 2007; Lee and Cho 2006; Prinzie and Van del Poel 2006]

\section{Operational CRM}

Operational CRM means that an ICT based support is provided for front office activities, including sales, service and support. Such support is intended to be used both in call/contact centres and by the sales staff in their customer contacts.

- One aspect of the relatively few articles on Operational CRM (see Table 1) concerns personalised customer service, mainly managed in contact centres [Koole 2004]. Personalised service also comprises real-time web interaction with sales representatives and support staff [Ohaegbu and Devgan 2000].

- An important goal for Operational CRM is to measure, improve and optimise the performance of customer services and the sales force. Sales force support and information is therefore necessary in order to develop sales and sales force management functions. Sales people are regarded as important information gatherers [Liu and Comer 2006] and an issue addressed is what happens once sales force automation (SFA) technology is adopted (Buehrer et al. 2005).

- Surprisingly few articles cover IT or CRM software, even though there are a few articles covering CRM packages in general [Light 2003] and internet based phone communication [Moon et al. 2000].

\section{Collaborative CRM}

The development of ICT has opened up new possibilities for communicating with the customers. In addition to personal contacts, telephone contacts and mails, it is now also possible to communicate through web sites, e-mail, selfservice telephone contacts, and SMS. Through this development, the use of different communication channels in a management strategy has become a branch of CRM. The Collaborative CRM concept covers this branch of CRM.

- The relatively few articles (see Table 1) on Collaborative CRM are dominated by the Internet and web channels. Internet marketing and online shopping call for shopping agents, product configurations, collaborative recommender systems, web complaint management, and general customisable services [Fan et al. 2004; Kruse and Bramham 2003; O'Leary et al. 2004]. On the other hand, all other kinds of non-commercial web services and information portals require web-based tools, web accessibility and web-enabled servicing in general [Yang et al. 2003].

- New additions to self-service channels are the mobile channels. Mobile computing and mobile equipment in general require development of mobile CRM, i.e. management of customer relations using mobile equipment [Camponovo et al. 2005; Shen and Lee 2000]

- Lastly, Collaborative CRM also relates to the field of elearning. Web-based learning platforms and mobile student information tracking systems are examples of article topics [Neville et al. 2005]

\section{SYSTEM ARCHITRECTURE}

\section{MVC ARCHITECTURE}

CRM system uses MVC (Model, View and Controller). MVC is a software architectural pattern for implementing user interfaces on computers. It divides a given application into three interconnected parts. This is done to separate internal representations of information from the ways information is presented to, and accepted from the user.

○ MVC stands for "Model view And Controller".

- The main aim of MVC Architecture is to separate the Business logic \& Application data from the USER interface.

- Different types of Architectures are available. These are 3-tier Architecture, N-tier Architecture, MVC Architecture, etc.

- The main advantage of Architecture is Reusability, Security and Increasing the performance of Application.

Model: Database operation such as fetch data or update data etc.

View: End-user GUI through which user can interact with system, i.e., HTML, CSS.

Controller: Contain Business logic and provide a link between model and view.

Let's understand this MVC concept in detail:

A. Model:

○ The Model object knows all about all the data that need to be displayed.

- The Model represents the application data and business rules that govern to an update of data.

- Model is not aware about the presentation of data and How the data will be display to the browser.

B. View:

CRM follows the MVC (Model-View-Controller) pattern and as such has the concept of views. Views are responsible for gathering and displaying data. There are a number of default views in Suite CRM. These include: 


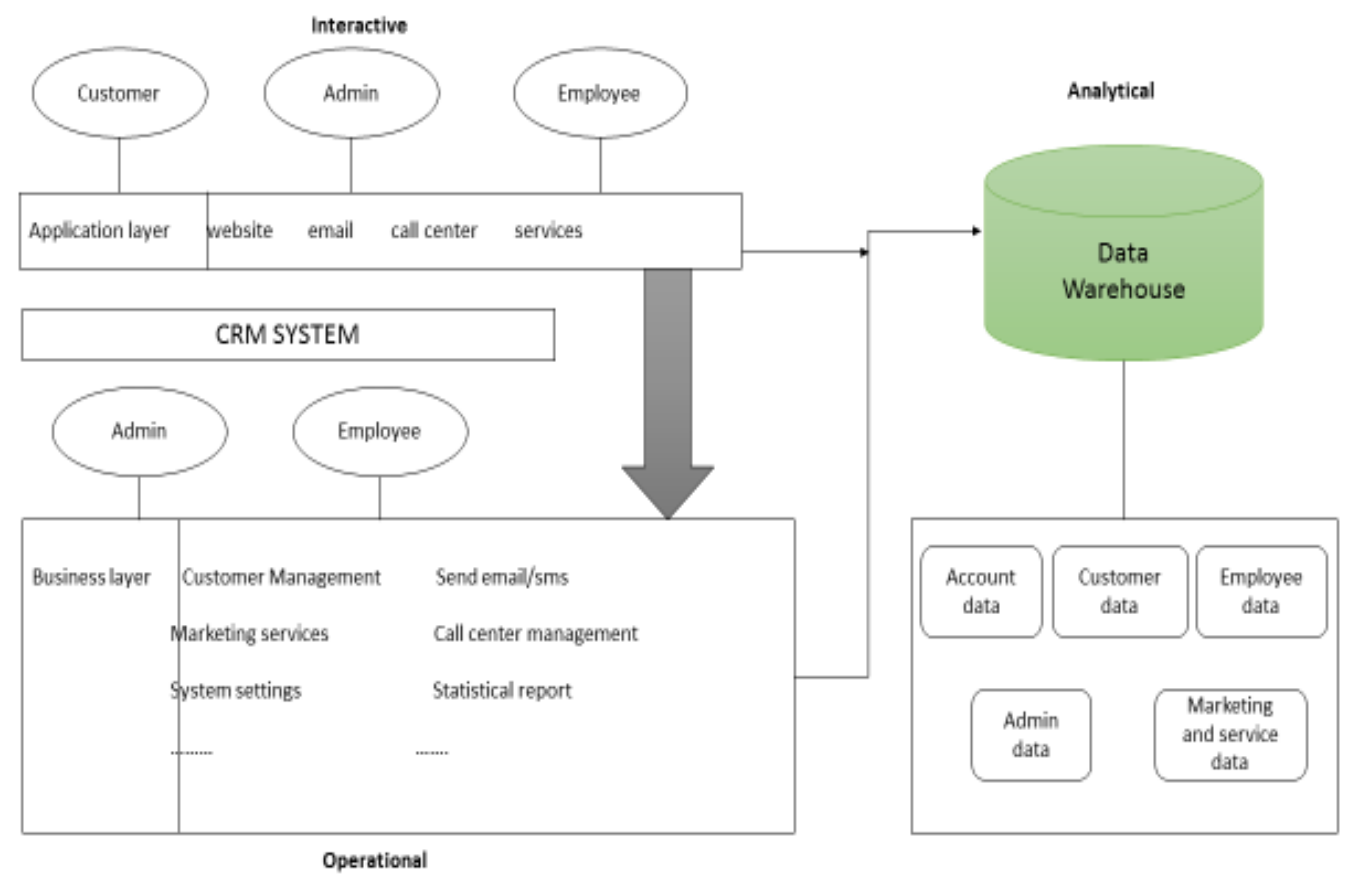

Fig 1. System Architecture

\section{ListView}

Displays a list of records and provides links to the EditViews and DetailViews of those records. The ListView also allows some operations such as deleting and mass updating records. This is (usually) the default view for a module.

\section{DetailView}

Displays the details of a single record and also displays subpanels of related records.

\section{EditView}

The EditView allows editing the various fields of a record and provides validation on these values.

The View represents the presentation of the application. View object refers to the model remains same if there are any modifications in the Business logic.

In other words, we can say that it is the responsibility of view to maintain consistency in its presentation and the model changes. Controller: Whenever the user sends a request for something, it always goes through Controller. A controller is responsible for intercepting the request from view and passes to the model for appropriate action. After the action has been taken on the data, the controller is responsible for directly passes the appropriate view to the user. In graphical user interfaces, controller and view work very closely together.

\section{METHODOLOGICAL LIMITATIONS}

The method used for this thesis has some limitations that could affect the result, or at least seem to have an impact. For starter, the low number of articles used and respondents from the interviews. The year when the articles and figures were published. The fact that there still is knowledge gaps within this area of expertise - there is no perfect answer nor approach which could be standardized and used for each and every organization. To be fair, there are a lot of different aspects that needs to be considered within a CRM, meaning that there are few articles exclusively focusing on the CRM as whole. This also gives the few articles written within this specific area a depth that could only be achieved through a broad empirical background. The number of interviews is low, that's why they are used as a supplement - to gain information from a different source. What could be worth, once again, to mention is that even thought it was only two participants, it was their company's complete IT-staff. The articles are year's apart showing the frequency of published works, but shows the reliability - the issues are still the same and there is still a struggle in generating an exact answer for this issue. Meaning that the issues are still current, but there are just few studies done within this topic. This makes a small but reliable platform for this thesis.

\section{MOST RECURRENT ISSUES}

From the research based on literature analysis and analysis of empirical observations done within these articles, one conclusion that could be drawn would be that there are a couple of recurrent issues. To bring such a huge system as a companywide system of CRM will leave its marks, and will most likely change the entire organizational infrastructure. This is independently of the fact that there are different kinds of systems for CRM due to, first of all, that there is no exact way of what the systems should contain in functions or technology and lastly that there is no exact measure of how much of the company it will include or where its precise focus should lie - either on the customers or even within the company itself. Just to point out, the focus with a system of CRM is of course on the customer relationship, but there are different ways to achieve this relationship and the two of them are either to focus on the customer and change accordingly to increase the meeting with the customer or to focus on the company and change it accordingly to achieve a better relationship with the customer. The only thing that is consistent is that there will always be a change in the organizational infrastructure.

\section{DISCUSSION}

CRM is relatively new and in the beginning the problems was in determining what features it would include and what area it would concentrate within. Hence, it has a huge selection of different variants of systems that focus on different angles. Today there are many systems and the different perspectives have contributed to a variety of systems that created a market where there can be found a system that suits one's business best. The problem is no longer in the system, but from what 
we can see in this thesis, it is due to non-static resources, such as the personnel and the customers. It will establish how much technology that needs to involved, while providing an opportunity to reflect on what departments it shall include, the most. Why not think of the company as whole, when adopting the system, are up for the management. To focus on a few departments in the beginning and then involve more and more could be a good way to secure a success. All focus lies within the department that are going to use it the most, and let them have all the consults and all help that they need to completely understand the system. There needs to be an awareness of the fact that if there is a change in the system, it will change the workflow which ultimately will change the organizational infrastructure and culture.

\section{ACKNOWLEDGMENT}

Apart from our own, the success of this report depends largely on the encouragement and guidelines of many others. We are especially grateful to our external guide Mr. Dheeraj Kohli, AUX services, USA who have provided all the guidance, expertise and encouragement. He spent his valuable time for us and we could do this because of his motivation. We express our heartfelt gratefulness to our internal guide Dr. Deepak A. Vidhate, HOD IT and Prof. Ms. Shruti S. Pophale, Project Coordinator and Asst. Prof. of Department of Information Technology, DVVPCOE for stimulating supervision whenever required during the entire project work. We are also thankful to the staff of Information Technology Department for their cooperation and support. We would like to put forward our heartfelt acknowledgement to all our classmates, friends and all those who have directly or indirectly provided their overwhelming support during our seminar work and the development of this report.

\section{REFERENCES}

[1] Chen, I. J., \& Popovich, K. "Understanding customer relationship management (CRM): People, process and technology" Business Process Management Journal, 9(5), 672- 688, 2003.

[2] Deepak A Vidhate, Parag Kulkarni, "Performance comparison of multiagent cooperative reinforcement learning algorithms for dynamic decision making in retail shop application", International Journal of Computational Systems Engineering, Inderscience Publishers (IEL), Volume 5, Issue 3, pp 169-178, 2019.

[3] Bain \& Company, "Insights - Management tools" Available at: http://www.bain.com/publications/articles/managementtools-customerrelationship-management.aspx, Accessed May 10, 2013.

[4] Bevan, K., Galbreath, J., Heth, M. "The great debate Justifying your investment in customer relationship management solutions" Available at: http://www.crmxchange.com, Accessed April 28, 2013.

[5] Deepak A Vidhate, Parag Kulkarni, "A Framework for Dynamic Decision Making by Multi-agent Cooperative Fault Pair Algorithm (MCFPA) in Retail Shop Application", Information and Communication Technology for Intelligent Systems, Springer, Singapore, pp 693-703, 2019.

[6] Fletcher, L. A. "Going beyond the buzzword: what exactly is CRM?" Learned Publishing, 14(3), 213-222, 2001.

[7] Deepak A Vidhate, Parag Kulkarni, "A Novel Approach by Cooperative Multiagent Fault Pair Learning
(CMFPL)" Communications in Computer and Information Science, Springer, Singapore, Volume 905, pp 352-361,2018.

[8] Payne, A., \& Frow, P."The role of multichannel integration in customer relationship management" Industrial Marketing Management, 33(6), 527-538,2004.

[9] Deepak A. Vidhate and Parag Kulkarni, "Expertise Based Cooperative Reinforcement Learning Methods (ECRLM)", International Conference on Information \& Communication Technology for Intelligent System, Springer book series Smart Innovation, Systems and Technologies (SIST, volume 84), Cham, pp 350-360, 2017.

[10] Payne, A., \& Frow, P “A strategic framework for customer relationship management", Journal of marketing, 167-176,2005.

[11] Deepak A. Vidhate and Parag Kulkarni "Innovative Approach Towards Cooperation Models for Multi-agent Reinforcement Learning (CMMARL) "International Conference on Smart Trends for Information Technology and Computer Communications Springer, Singapore, pp. 468-478, 2016.

[12] Ryals, L., \& Knox, S. "Cross-functional issues in the implementation of relationship marketing through customer relationship management" European Management Journal, 19(5), 534-542,2001.

[13] Xu, Y., Yen, D. C., Lin, B., \& Chou, D. C. "Adopting customer relationship management technology" Industrial management \& data systems,102(8), 442452,2002 .

[14] Deepak A Vidhate and Parag Kulkarni, "Enhanced Cooperative Multi-agent Learning Algorithms (ECMLA) using Reinforcement Learning" International Conference on Computing, Analytics and Security Trends (CAST), IEEE Xplorer, pp 556 - 561, 2017

[15] Ngai E.W.T. "Customer relationship management research (1992-2002)" An academic literature review and classification. Marketing Intelligence \& Planning, 23(6) pp. 582-605,2005.

[16] Osarenkhoe A. "Customer-Centric Strategy: A longitudinal study of implementation of a customer relationship management solution" International Journal of Technology Marketing, 1(2), pp. 115-143,2006.

[17] Deepak A Vidhate, Parag Kulkarni, "Exploring Cooperative Multi-agent Reinforcement Learning Algorithm (CMRLA) for Intelligent Traffic Signal Control", Smart Trends in Information Technology and Computer Communications. SmartCom 2017, Volume 876, pp 71-81,2018.

[18] Özgener S. and Iraz R. "Customer relationship management in small-medium enterprises: The case of Turkish tourism industry" Tourism Management 27, pp. 1356-1363, 2006.

[19] Lenskold J. D. "Customer-Centric Marketing ROI" Marketing Management 13(1), pp. 26-31, 2004.

[20] Cleary C. "Strategic issues in customer relationship management CRM) implementation" Business Process Management Journal 9(5), pp. 592 - 602, 2003. 